\title{
Evolution des pratiques de traction animale et conséquences sur la durabilité des systèmes de culture
}

\author{
E. Vall ${ }^{1 *}$ A.L. Dongmo Ngoutsop ${ }^{2}$ \\ T. $\mathrm{Ndao}^{3}$ I. Ilboudo ${ }^{4}$
}

\section{Mots-clés}

Energie animale - Culture attelée Afrique occidentale - Afrique centrale.

\begin{abstract}
Résumé
Dans les systèmes de culture des zones de savane de l'Afrique de l'Ouest et du Centre, la traction animale occupe une place déterminante pour renforcer la capacité $d^{\prime}$ intervention des producteurs lors des étapes clés du calendrier agricole: préparation des terres, semis, désherbage, transport. Ces dix dernières années, trois facteurs ont déterminé les évolutions des pratiques: i) le démantèlement des services d'appui publics a rendu plus difficile l'acquisition et l'entretien des attelages, ii) I'augmentation de la pression foncière en zone rurale a renforcé les synergies agriculture élevage et induit des pratiques $d^{\prime}$ intensification par le travail (mécanisation des désherbages...), iii) la diffusion des herbicides a provoqué un repli du labour. Les conséquences agronomiques de ces évolutions sur la durabilité des systèmes de production sont parfois positives (amélioration de la flexibilité du travail, de la production et des revenus financiers des ménages), parfois négatives (marginalisation du rôle de l'élevage dans les transferts de fertilité, dégradation du semis mécanique, dégradation des itinéraires techniques, déforestation...). Des propositions d'amélioration de la technique sont suggérées.
\end{abstract}

\section{INTRODUCTION}

Ces cinquante dernières années, la traction animale a été une innovation majeure dans l'agriculture familiale des savanes d'Afrique de l'Ouest et du Centre. Elle fût introduite pour soutenir le développement des cultures industrielles (coton, arachide, riz) en contribuant à accroître la productivité du travail et à stimuler les synergies entre l'élevage et l'agriculture (19). Elle a contribué à une transformation rapide des systèmes de production autrefois peu monétarisés et caractérisés par une dissociation marquée entre l'élevage et l'agriculture (17). Cette région compte aujourd'hui 2,5 millions d'attelages, cinq fois plus qu'en 1965 (13). La traction animale est utilisée par la majorité des paysans, soit comme propriétaires, soit comme locataires d'attelages dans des exploitations familiales cultivant 3 à 20 ha (34).

Depuis une dizaine d'années, cette région du monde est en proie à de nombreuses mutations liées à la libéralisation de l'économie, au

\footnotetext{
1. Cirad, département Emvt, TA30/A, campus international de Baillarguet, 34398 Montpellier Cedex 5, France

2. Irad, station polyvalente, BP 415, Garoua, Cameroun

3. Isra, Cnra Bambey, BP 53, Bambey, Sénégal

4. Drahrh, BP 26, Fada Ngourma, Burkina Faso

* Adresse actuelle : Cirdes, BP 454, Bobo-Dioulasso 01, Burkina Faso
}

désengagement des Etats, au mouvement de professionnalisation des producteurs, aux processus de décentralisation, à l'augmentation de la pression foncière dans les zones rurales, à l'accroissement de la demande en denrées agricoles des zones urbaines, au progrès des techniques (22). Ces mutations ont des conséquences multiples sur les stratégies de production et d'organisation du travail dans les exploitations. La place et le rôle de l'animal de trait dans les systèmes de culture et les systèmes de production sont affectés par ces changements.

Cet article passe en revue l'évolution des pratiques de traction animale ainsi que leurs conséquences sur la durabilité des systèmes de production et présente une série de propositions d'améliorations techniques.

\section{DIVERSITE DE LA PLACE ET DU ROLE DE LA TRACTION ANIMALE}

\section{La traction animale dans les zones écologiques et les calendriers agricoles}

La mécanisation à traction animale intervient pour la préparation des sols (labour, scarifiage...), le semis, la maîtrise des adventices (labour, sarclage, buttage), la gestion de l'eau en début et fin de cycle (labour, scarifiage, buttage), l'entretien de la fertilité (enfouissement matière organique, production de fumure) et les 
multiples opérations de transport ${ }^{1}$ (34). Les techniques varient selon les zones agroécologiques et selon les systèmes de culture. Les systèmes coton/maïs/sorgho/arachide et arachide/mil/sorgho des zones semi-arides et subhumides représentent les situations où la traction animale a connu le plus fort développement (tableau I).

En zone semi-aride, la pluviosité faible, aléatoire, répartie sur une courte période est une contrainte majeure qui limite la diversité des cultures pluviales (figure 1). La rapidité d'intervention est déterminante pour la réussite des cultures tant au semis qu'au désherbage. Les travaux de préparation, effectués sur des sols le plus souvent sableux, sont superficiels et ne nécessitent pas une importante force de traction. Les exploitations sont généralement bien équipées en outils de désherbage (houes occidentale, Sine, Manga...) car la maîtrise des adventices est déterminante pour le succès de la campagne. Le soulevage de l'arachide est mécanisé lorsque les sols sont sableux. Il est réalisé soit au moyen d'une lame souleveuse spécifique montée sur le bâti d'un polyculteur (Sine), soit à la charrue en ôtant le versoir. C'est la seule opération de récolte mécanisée. Les techniques de transport à énergie animale sont plus diversifiées que dans les zones subhumides, probablement parce que la mobilité contribue à la sécurisation des systèmes de production dans des zones où les ressources sont éparses.

Dans les zones subhumides, la pluviométrie, plus élevée, autorise une gamme de cultures élargie (coton, maiis, sorgho, arachide...) et permet un étalement des dates de semis, mais la maîtrise des

${ }^{1}$ La fonction de transport ne sera pas développée dans cette communication. Pour cela on se réfèrera à l'article de Paul Starkey. adventices demande plus d'efforts (figure 1). Le labour d'enfouissement des adventices est une opération très importante pour réussir les semis du coton et du maïs. Au Nord-Cameroun jusqu'en 1999, 76 p. 100 de la surface cotonnière était labourée à plat. Les surfaces restantes étaient semées sans préparation avec épandage d'herbicides totaux. Dans l'est du Burkina Faso, environ 75 p. 100 des exploitations labourent les parcelles de céréales et de cotonnier en préparant des billons. Dans les zones subhumides, le désherbage mécanique correspond à une suite d'interventions qui commence par le labour, suivi d'un à trois sarclages (selon les cultures et les états d'enherbement; voir plus loin) et d'un buttage pour le coton et le maiis. Avec le buttage, trois objectifs sont poursuivis : i) éviter les excès d'eau au cours du cycle, ii) désherber, iii) recouvrir l'urée ; on recommande de butter le coton et le maïs, 30 à 40 jours après la levée. En raison d'une pression plus forte des adventices, le désherbage mécanique nécessite des outils plus robustes et des attelages plus puissants que dans les zones semi-arides. Au Nord-Cameroun, les sarclages restent le plus souvent manuels (plus de 40 p. 100 de la surface cotonnière sarclée mécaniquement), mais la mécanisation progresse, notamment en zone subhumide, et les travaux de buttage sont le plus souvent mécanisés (à plus de 80 p. 100 ; 23). De plus en plus souvent, les herbicides sont utilisés en complément, en relais et parfois en substitution de la mécanisation (voir plus loin).

\section{Critères de choix des animaux et conduite des attelages}

Dans les zones semi-arides, les ânes et les chevaux trouvent un environnement adapté à leur physiologie (climat sec et absence de

\section{Tableau I}

Utilisation de la traction animale selon les systèmes de culture et les zones agroécologiques en Afrique subsaharienne

\begin{tabular}{|c|c|c|c|c|}
\hline Systèmes de culture & $\begin{array}{l}\text { Zones arides } \\
<400 \mathrm{~mm}\end{array}$ & $\begin{array}{l}\text { Zones semi-arides } \\
\text { 400-800 mm }\end{array}$ & $\begin{array}{l}\text { Zones subhumides } \\
\text { 800-1 } 200 \mathrm{~mm}\end{array}$ & $\begin{array}{l}\text { Zones humides } \\
>1200 \mathrm{~mm}\end{array}$ \\
\hline $\begin{array}{l}\text { Oasien et } \\
\text { Maraîchage }\end{array}$ & $\begin{array}{l}\text { Exhaure, bât, charrette } \\
\text { Ane, cheval, dromadaire }\end{array}$ & $\begin{array}{l}\text { Exhaure, bât, charrette } \\
\text { Ane, cheval (dromadaire) }\end{array}$ & Transport, fumure & Transport, fumure \\
\hline Mil/sorgho & - & $\begin{array}{l}\text { Travail du sol en sec } \\
\text { (labour), sarclage, } \\
\text { charrette } \\
\text { Ane, cheval (bovin) }\end{array}$ & $\begin{array}{l}\text { Labour, sarclage, } \\
\text { charrette } \\
\text { Bovin (âne) }\end{array}$ & - \\
\hline $\begin{array}{l}\text { Arachide/mil/ } \\
\text { sorgho }\end{array}$ & - & $\begin{array}{l}\text { Semis, sarclage, } \\
\text { soulevage, charrette } \\
\text { Cheval, âne (bovin) }\end{array}$ & $\begin{array}{l}\text { Labour, semis, sarclage, } \\
\text { soulevage, charrette } \\
\text { Bovin (âne) }\end{array}$ & - \\
\hline $\begin{array}{l}\text { Coton/maïs/ } \\
\text { sorgho/arachide }\end{array}$ & - & $\begin{array}{l}\text { Labour, sarclage, } \\
\text { buttage, charrette } \\
\text { Ane (bovin) }\end{array}$ & $\begin{array}{l}\text { Labour, sarclage, } \\
\text { buttage, charrette } \\
\text { Bovin (âne) }\end{array}$ & $\begin{array}{l}\text { Labour, sarclage, } \\
\text { buttage } \\
\text { Bovin taurin }\end{array}$ \\
\hline Riziculture irriguée & $\begin{array}{l}\text { Labour, charrette } \\
\text { Cheval, âne, bovin }\end{array}$ & $\begin{array}{l}\text { Labour, charrette } \\
\text { Cheval, âne, bovin }\end{array}$ & - & - \\
\hline $\begin{array}{l}\text { Cultures de décrue } \\
\text { (riz, sorgho) }\end{array}$ & - & Marginale & Marginale & - \\
\hline Cultures de bas-fonds & - & $\begin{array}{l}\text { Labour } \\
\text { Bovin }\end{array}$ & $\begin{array}{l}\text { Labour } \\
\text { Bovin }\end{array}$ & $\begin{array}{l}\text { Labour } \\
\text { Bovin }\end{array}$ \\
\hline $\begin{array}{l}\text { Tubercules } \\
\text { (igname, manioc...) }\end{array}$ & - & - & $\begin{array}{l}\text { Labour, buttage } \\
\text { Bovin }\end{array}$ & $\begin{array}{l}\text { Labour, buttage } \\
\text { Bovin }\end{array}$ \\
\hline $\begin{array}{l}\text { Cultures pérennes } \\
\text { (canne, palmier) }\end{array}$ & - & - & - & $\begin{array}{l}\text { Charrette } \\
\text { Taurin }\end{array}$ \\
\hline
\end{tabular}

Sur fond blanc sont indiquées les zones d'utilisation intense de la traction animale 


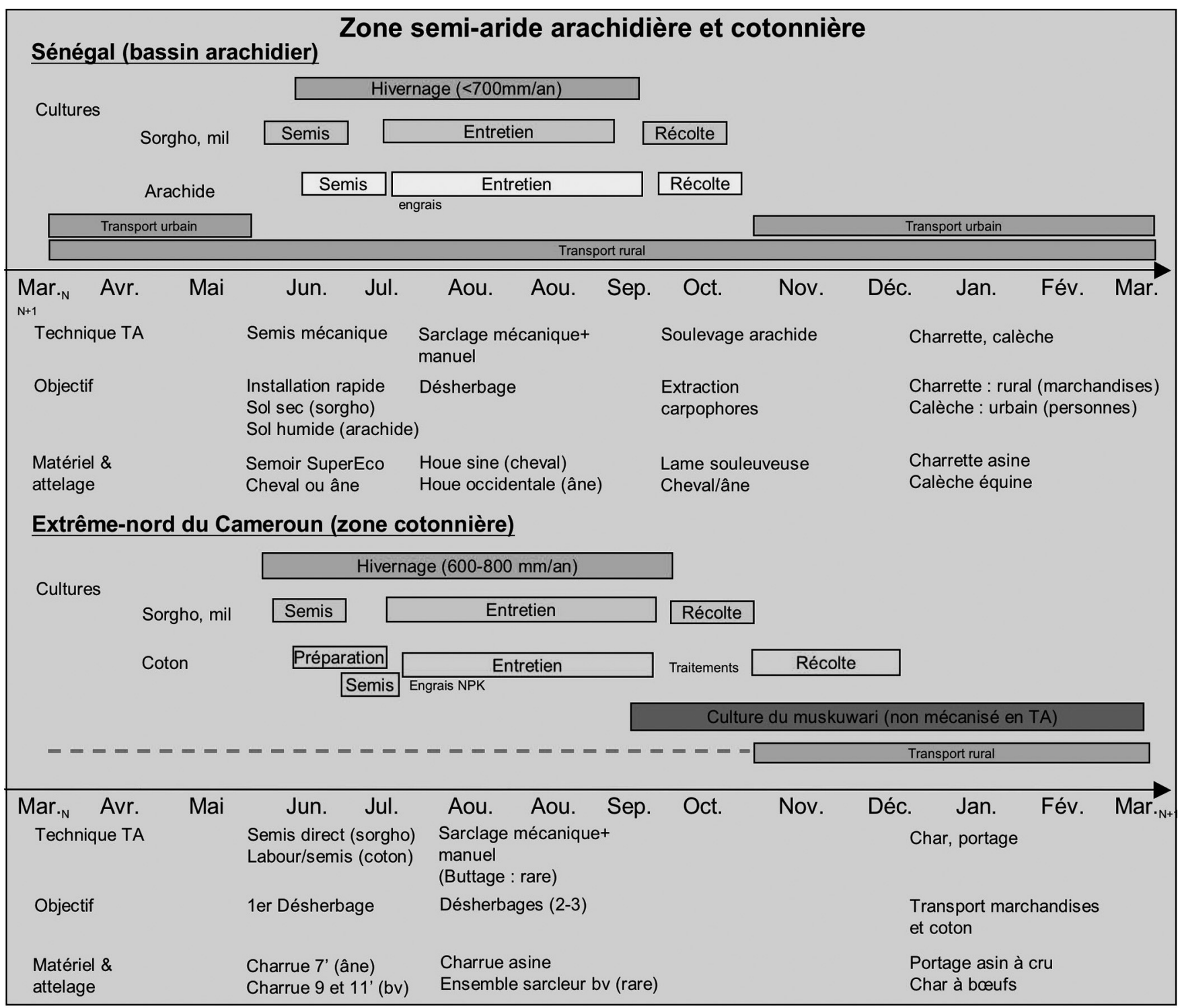

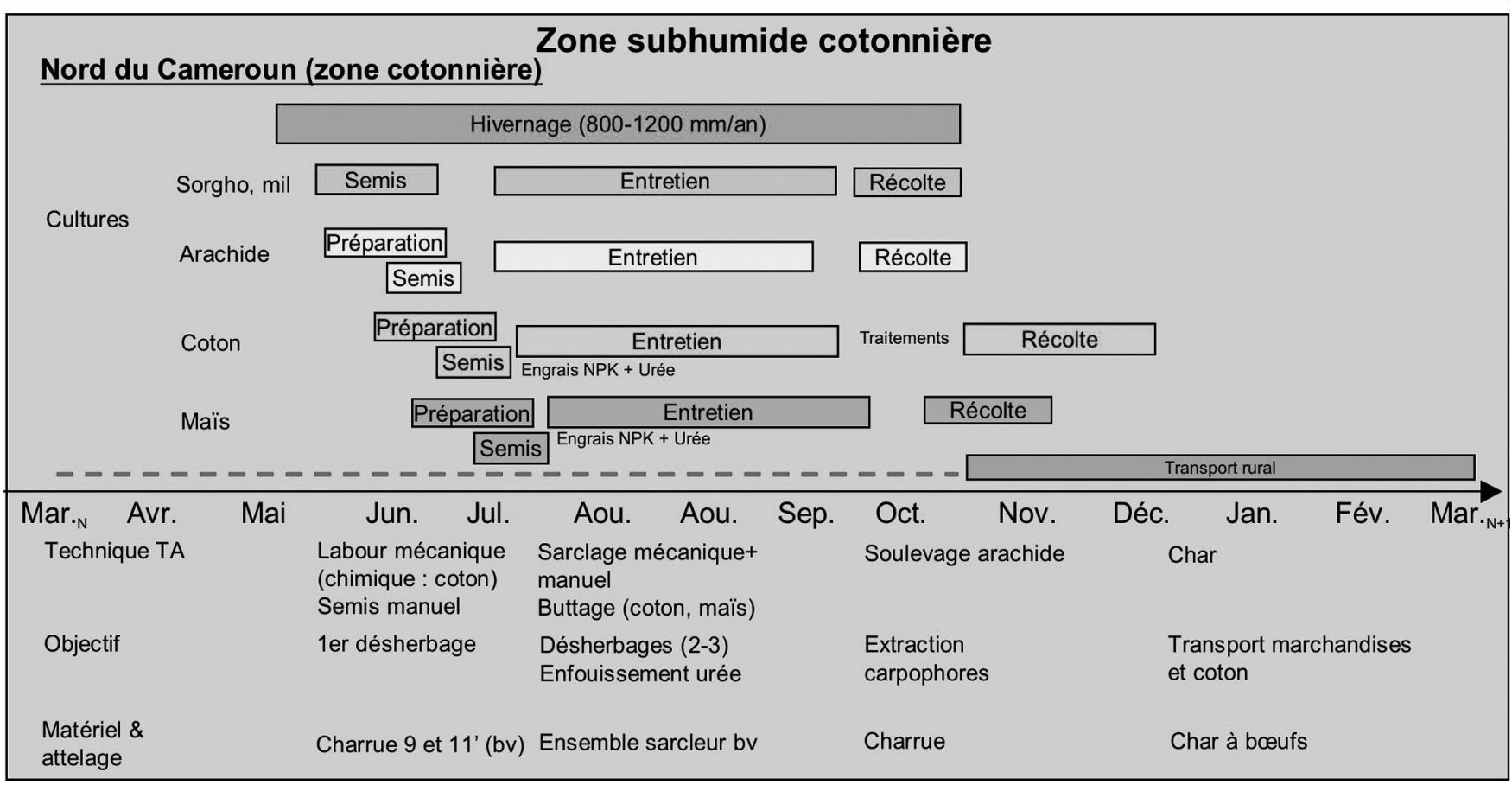

Figure 1 : place et rôle de la traction animale dans les calendriers agricoles des zones étudiées (la figure ne prend en compte que les cultures principales concernées par la mécanisation). 
trypanosomose). C'est sans doute une des raisons ayant conduit les paysans à préférer ces espèces. Se sont de plus des attelages légers et rapides, qualités importantes dans des systèmes de culture où les interventions doivent suivre au plus près les rares pluies utiles. Enfin ces espèces sont adaptées au portage et aux longues distances, comme l'exige de nombreuses tâches quotidiennes dans les zones sèches (bois de feu, eau).

L'âne est économique à l'achat $\left(40000 \mathrm{Fcfa}^{1}\right)$ et à l'entretien $(<5000 \mathrm{Fcfa} / \mathrm{an})$. Il se contente d'une nourriture peu recherchée. Ses besoins en énergie et en eau sont modestes (29). Ces caractéristiques en font le parfait animal de travail des paysans modestes en zone aride. Cependant sa capacité de travail est limitée (puissance $100 \mathrm{w} ; 24$ ) et il ne peut effectuer que des travaux du sol légers et superficiels. Elle est néanmoins souvent suffisante sur les sols arénacés, fréquents en zone aride, où les travaux agricoles nécessitent surtout des façons culturales superficielles. Parfois, pour compenser leur faible puissance, les paysans utilisent plusieurs ânes en relais. En général, ils attèlent indifféremment les mâles et les femelles car leur capacité de travail est comparable (8, 24). Les carrières des ânes comme celles des chevaux sont souvent longues, car, contrairement aux bovins, ils ne sont pas réformés en boucherie.

Les chevaux de trait sont souvent des poneys mâles (métis Mbayar x Mpar au Sénégal, Museys au Nord-Cameroun), plus économiques et rustiques que les races de grands chevaux. Un poney coûte entre 600000 et $70000 \mathrm{Fcfa}(3,21,33)$. L'alimentation du poney est exigeante en qualité et en quantité, donc coûteuse. (Exemple de ration pour un travail moyen : $4,5 \mathrm{~kg}$ de $\mathrm{MS} / \mathrm{j}$ de fane d'arachide + $1 \mathrm{~kg}$ de MS/j de sorgho ; 19.) Sa puissance de traction est élevée (250 w en moyenne ; 24). L'attelage de jument est peu fréquent. Il semble qu'en Afrique noire les paysans soient très réticents à soumettre les femelles d'espèces nobles et coûteuses au travail (chevaux et bovins) car cela est jugé peu compatible avec la fonction de reproduction.

Dans les zones subhumides, les paysans utilisent principalement des paires de zébus mâles, le plus souvent non castrés, attelés par des jougs de garrots. Avec un poids vif de 350 à $500 \mathrm{~kg}$ une paire développe aisément une puissance de 600 w (24). Dressés vers l'âge de 3 ans, ils ont généralement des carrières assez courtes de 4 à 5 années. Au Nord-Cameroun, un taurillon de 3 ans de $80000 \mathrm{Fcfa}$ atteint $180000 \mathrm{Fcfa}$ en fin de carrière. Le bovin de trait est une forme d'épargne avec un taux d'intérêt annuel compris entre 10 et 15 p. 100, ce qui a contribué à l'engouement des paysans pour ce type d'attelage $(2,4,33)$. Dans ces zones, l'alimentation pose moins de difficultés. Le stockage de pailles et de fanes ainsi que de tourteau de coton est fréquent en vue de l'affouragement de fin de saison sèche. Le suivi sanitaire doit être plus rigoureux qu'en zone aride car la pression parasitaire est forte (glossines, tiques...). Cependant, les défrichements agricoles détruisent les habitats des parasites et font reculer certaines maladies (trypanosomoses).

\section{Préparation du sol à la charrue, emblème de la culture attelée en zone cotonnière}

Dans les zones semi-arides, les céréales sont le plus souvent semées sans préparation du sol immédiatement après la première pluie utile. Le semis du coton intervient ensuite, après plusieurs pluies utiles, si bien qu'un labour est nécessaire pour enfouir les adventices. Cependant, le labour est superficiel et les sillons le plus souvent disjoints. En espaçant les passages de l'outil le paysan gagne du temps. Le travail du sol limité à la ligne de semis vise à faciliter l'infiltration de l'eau et la germination $(4,15,33,37)$.

\footnotetext{
$1 €=655 \mathrm{Fcfa}$
}

Dans les zones cotonnières subhumides, la fonction principale du labour est le désherbage avant les semis (coton, maïs, arachide, sorgho). Le labour est effectué après la première ou la seconde pluie utile, juste avant le semis. Les bovins sont attelés à des charrues lourdes (9 à 11 inches) et les ânes à des charrues légères (6 à 7 inches). Le travail du sol est peu profond $(<15 \mathrm{~cm})$. Pour gagner du temps, les paysans ne labourent pas toute la surface du champs, soit en écartant les passages de la charrue (1,5 x largeur du sillon) dans le cas d'un labour à plat comme au Cameroun, soit en pratiquant le billonnage (retour de la charrue sur la même ligne) comme au Burkina Faso. Le labour mécanique augmente fortement la productivité du travail par rapport au labour manuel et permet d'augmenter significativement les emblavements $(19,34)$. Pour cette raison, la charrue est devenue l'emblème de la traction animale en zone cotonnière ; les bovins de trait ont été rebaptisés bœufs de labour.

\section{Semis mécanique, une fonction spécifique de la traction animale en zone semi-aride}

Le semis mécanique est parfaitement au point pour les céréales (mil, sorgho, maïs), les légumineuses (arachide, niébé), le soja et le sésame, moyennant l'usage de disques adaptés. Pour le coton, il requiert des semences délintés, ce qui a freiné sa vulgarisation. Il permet de semer rapidement dans des conditions d'humidité optimale, ce qui est déterminant en zone semi-aride, et d'obtenir une bonne densité de culture lorsque la technique est maîtrisée (encadré 1) et lorsque les sols sont légers (sableux). Au Sénégal, ces conditions de réussites, auxquelles il faut ajouter l'organisation de la filière arachide, favorisent l'engouement des paysans pour cette pratique qui concerne 90 p. 100 des parcelles de céréales et d'arachide (9). Ailleurs ces conditions n'ont pas été réunies et les semis restent le plus souvent manuels. C'est le cas en Afrique centrale, où la technique n'a pas été vulgarisée (33). En Afrique de l'Ouest, la technique progresse dans certaines zones de production du maïs (Mali Sud) et pour certaines productions à haute valeur ajoutée (comme le souchet au Niger) pour augmenter les densités de semis et les rendements.

Encadré 1

\section{LE SEMIS DE L'ARACHIDE AU SÉNÉGAL}

Le coût du semis est très élevé en raison du prix de la semence et de la dose requise $(60 \mathrm{~kg}$ graines/ha soit entre $1 / 5$ et $1 / 6$ de la production, pour une densité de semis recommandée entre 110000 et 170000 pieds/ha selon les variétés). II est impératif de ne pas le rater. C'est pourquoi l'arachide est semée après une pluie de $25 \mathrm{~mm}$. Le mil et le sorgho exigent des doses de semences plus faibles (3 à $10 \mathrm{~kg} / \mathrm{ha}$ ). Les paysans prennent le risque de semer le mil en sec et le sorgho sur de petites pluies pour gagner du temps, quitte à resemer plus tard en cas de mauvaise levée $(3,9,12)$. Ils utilisent des semoirs Super-Eco monorang introduits dans les années 1920. Sur sols sableux, cette opération requiert peu de force de traction (20 à 30 daN). La distribution d'un disque varie en fonction du nombre, de la forme et de la taille des crans à adapter aux graines à semer. Comme les variétés d'arachide ont des tailles de graines variées, plusieurs disques sont recommandés pour des interlignes de semis précis : le 24 crans pour les variétés hâtives à petite graine (35 à $40 \mathrm{~g}$ pour 100 graines) à $0,45 \mathrm{~m}$ d'interligne, le 30 crans pour les variétés tardives ou semi-tardives à graines moyennes (45 à 55 g pour 100 graines) à 0,5 ou 0,6 m d'interligne, le 20 crans pour les arachides de bouche ( 65 à $85 \mathrm{~g}$ pour 100 graines) à 0,6 m d'interligne (Havard, 1988, Mach. agric. trop). 


\section{Entretien des cultures mécanique : une technique en cours d'adoption}

Partout, les désherbages sont un goulot d'étranglement dans le calendrier agricole de juillet à août. C'est une opération très consommatrice de main d'œuvre lorsqu'elle est manuelle (21 à $35 \mathrm{j} /$ ha/actif/passage ; 31). Les chantiers avancent lentement, ce qui entretient les désherbages tardifs avec pour conséquence des pertes de production et des abandons de parcelles envahies par les adventices. (Les abandons s'inscrivent souvent dans une stratégie de sur-emblavement par rapport à la force de travail disponible sur l'exploitation, qui a pour but soit de sécuriser la production en cas de pluviosité insuffisante, soit d'occuper l'espace cultivable dans des contextes de forte emprise agricole.) Dans les zones subhumides cotonnières, le premier sarclage est généralement tardif (25 à 30 jours après le semis) car, tant que les labours et les semis du coton et du maïs ne sont pas achevés, ils restent prioritaires sur le désherbage de l'arachide et du sorgho (figure 1). Pour étaler les travaux de désherbages, certains paysans qui ne disposent pas d'une main d'œuvre importante fractionnent les semis d'une même culture en deux vagues $(15,31)$. Le coton, dont la croissance est lente par rapport aux adventices, est sarclé jusqu'à trois fois, puis il est butté, ce qui fait avec le labour quatre à cinq désherbages (sans compter les applications d'herbicides). Le maïs est sarclé deux fois puis butté (il reçoit aussi des herbicides). L'arachide et le sorgho sont sarclés une fois, parfois deux, mais ne sont pas buttés (pas d'apport d'urée en milieu de cycle).

Malgré son caractère fastidieux et éreintant, le désherbage manuel demeure plus fréquent que le sarclage mécanique, notamment dans les zones semi-arides où il fait souvent appel à des travaux communautaires. Le désherbage manuel permet aussi de préserver des plantes à vertus pharmaceutiques où alimentaires, ce qui est parfois recherché sur les champs de case (31).

Le désherbage mécanique requiert un semis en ligne et des interlignes réguliers. Beaucoup plus rapide que le désherbage manuel, il est néanmoins toujours suivi d'un travail de finition à la main entre les poquets et sur les bords des rangs. Sur cotonnier, la durée du sarclage, finitions comprises, est divisée par trois (de 21 à $7 \mathrm{j} / \mathrm{ha} / \mathrm{actif}$; 14). Facile à mettre en ouvre sur des sols légers, il est problématique dans des sols plus lourds. Au Sénégal, la houe occidentale et la houe Sine sont bien adaptées aux sols sablonneux et à la capacité de traction des équidés $(3,9,12)$. Dans l'Extrême-Nord Cameroun, les outils s'enfoncent dans des sols détrempés souvent argileux, ce qui rend les attelages asins inopérants (31). Plusieurs modèles de sarcleuses ont été testés par la recherche, sans grands résultats (27). Les paysans préfèrent sarcler avec une charrue en effectuant un double passage par rang.

Les freins à l'adoption du désherbage mécanique sont donc nombreux : pratiques des cultures intercalaires et des associations, sols trop lourds, adventices se prêtant mal à la mécanisation, comme, par exemple, Commelina spp. pour qui le travail du sol tend à favoriser la germination des graines et à multiplier les bouturages des stolons sectionnés (14), prix élevés des matériels ${ }^{1}$, indispensables travaux de finitions manuels entre les lignes et les poquets rendant impossible la mécanisation complète du désherbage.

Aujourd'hui, la mécanisation du désherbage est plus courante en Afrique de l'Ouest qu'en Afrique centrale car les conditions techniques et économiques ont favorisé sa diffusion. Dans certaines zones subhumides de l'Afrique centrale, comme le Nord-

\footnotetext{
${ }^{1}$ En Afrique centrale, les outils vulgarisés comportent des dents en aciers spéciaux pour travailler dans des sols lourds (14). Bien qu'étant conçus comme des ensembles adaptables sur le bâti des charrues, ils restent chers et diffusent peu comparativement à Afrique de l'Ouest où les forgerons maitrisent la fabrication de sarcleuses simples et très économiques.
}

Cameroun, les paysans sont équipés de charrues et reportent leur effort d'investissement sur ce type de matériel. Les déterminants en cause sont discutés plus loin.

\section{- EVOLUTION DE L'ENVIRONNEMENT SOCIOTECHNIQUE ET SES EFFETS SUR LES PRATIQUES DE TRACTION ANIMALE}

\section{Evolutions en cause}

\section{Désengagement de l'Etat du secteur rural et recomposition des services d'appui}

Avant la libéralisation du secteur agricole, les services d'appui à la traction animale étaient assurés par les sociétés publiques et parapubliques de développement et d'encadrement agricole : crédits, fourniture d'équipements et d'animaux dressés, suivi sanitaire, aliments concentrés... Avec le désengagement des Etats des filières agricoles industrielles, ces services doivent se privatiser et s'insérer dans une logique économique pour dégager une rentabilité suffisante. Force est de constater que si, pour les artisans forgerons et les commerçants de bétail, la transition s'est bien passée, elle est beaucoup plus périlleuse pour les nouveaux prestataires de services financiers, d'appui/conseil et les vétérinaires. Ils ont du mal à adapter leur offre de services à la demande et ont tendance à déserter les zones rurales pour les marchés de services urbains et périurbains plus actifs (34). Les paysans doivent adapter leurs pratiques d'acquisition mais aussi d'entretien des attelages et des outils.

\section{Augmentation de la population et de la pression foncière en zone rurale}

L'accroissement de la population rurale touche l'ensemble de l'Afrique subsaharienne (22). Il entretient une augmentation de la pression foncière dans les zones agricoles et des flux de producteurs des régions peuplées $\left(>80 \mathrm{hab} . / \mathrm{km}^{2}\right)$, souvent caractérisées par une faible pluviosité $(<800 \mathrm{~mm} / \mathrm{an})$, vers des régions soudaniennes plus arrosées $(>1000 \mathrm{~mm} / \mathrm{an})$ disposant d'importantes ressources en terre car moins peuplées $\left(<20 \mathrm{hab} . / \mathrm{km}^{2}\right)$. Lorsque l'emprise agricole dépasse un certain seuil, la durée des jachères se réduit et l'on passe à la culture continue. Aussi la mécanisation devient nécessaire pour maitriser les adventices, gérer l'eau à la parcelle (infiltration, drainage) et enfouir la matière organique. Dans les zones subhumides, aux pratiques de labour à la charrue s'ajoute peu à peu la technique de désherbage mécanique, mais aussi de buttage et parfois de semis mécanisé (cas du Mali).

\section{Diffusion des herbicides dans les zones cotonnières}

Depuis 10 ans, le Nord-Cameroun est pionnier en matière de diffusion des herbicides (31). Actuellement, 71 p. 100 de la surface cotonnière reçoit un traitement herbicide (23). Le coût modéré des traitements a été un élément déterminant de l'adoption des herbicides (4 $800 \mathrm{Fcfa} / \mathrm{ha}$ pour le Diuron, $3300 \mathrm{Fcfa} / \mathrm{ha}$ pour le glyphosate formulé à $360 \mathrm{~g} / \mathrm{l}, 3600 \mathrm{Fcfa} / \mathrm{ha}$ pour le paraquat). Les herbicides employés contrôlent les adventices lors des premiers stades de développement de la culture, au moment où les paysans ont peu de temps disponible pour le sarclage. La Société de développement du coton (Sodécoton) vulgarise un traitement effectué au semis associant un herbicide total non sélectif qui permet de détruire les mauvaises herbes levées (paraquat ou glyphosate) à un herbicide sélectif du coton qui contrôle les adventices à la germination (Diuron). Les cultures intensives du maïs surtout et 20 p. 100 des surfaces de sorgho reçoivent un traitement herbicide post-semis/pré-levé à l'atrazine. Les paysans apprécient l'efficacité de ce produit sur Commelina spp. et son prix très modéré. Une majorité d'agriculteurs combine l'application d'atrazine sur maïs à un herbicide total. Ils combinent aussi les désherbages chimiques et 
mécaniques, ce qui a une incidence sur les modalités de préparation de sol et sur la conduite des itinéraires techniques (14).

\section{Effets sur les pratiques}

\section{Diversification des modes d'accès à la traction animale face à la pénurie de crédit}

Le coût d'une paire de taurillons peut atteindre 40 p. 100 du revenu annuel d'une jeune exploitation. En l'absence de "crédits animaux », jugés trop risqués par les services financiers, l'achat d'un attelage est donc une opération difficile qui repose sur la capacité d'autofinancement du ménage. Aujourd'hui, l'offre de crédits équipements s'effondre quand elle n'est plus sécurisée par le prélèvement des annuités à la vente du coton $(35,37)$. Les taux d'intérêts élevés pratiqués par la microfinance ne sont pas compatibles avec la rentabilité à court terme de la traction animale. Pour l'instant les conditions ne sont pas réunies pour développer un marché sécurisé de crédits à la traction animale en dehors des sociétés cotonnières. Ces difficultés ont stimulé l'essor des attelages économiques (asins, équins), mais aussi les pratiques de location, surtout dans les zones peu équipées (2, 3, 4). Dans l'Extrême-Nord Cameroun, la traction asine a connu un fort engouement dès la fin des années 1980, en raison d'une augmentation subite du prix du bétail (33). Aujourd'hui, elle domine dans la partie semi-aride du bassin cotonnier où les exploitations sont petites et modestes. Les jeunes paysans achètent des taurillons un par un et constituent une paire en deux ans qui devient opérationnelle quatre ans après le premier achat... La location payante est la formule la plus courante (15000 à $20000 \mathrm{Fcfa} /$ ha labouré). Elle est payable soit au comptant, soit à la commercialisation du coton, ou en main-d'œuvre (désherbage, récolte). Parfois deux propriétaires possédant un seul bovin associent leurs animaux pour une campagne. Les plus indigents s'engagent comme «bouviers » chez un propriétaire d'attelage (trois jours chez le patron contre un jour chez soi). Mais les règles de la location donnent toujours la priorité au propriétaire, ce qui fait systématiquement prendre du retard aux locataires et porte atteinte au plein succès de leur campagne agricole (dans les zones où l'offre de location est faible le retard peu atteindre 15 jours...).

\section{Emergence des forgerons et adaptation des matériels}

Les artisans forgerons ont progressivement investi le marché des équipements de traction animale, se substituant à l'industrie dans de nombreux pays (20). Le plus souvent, ils ont commencé par la production de pièces de rechange, puis sont passés à la fabrication d'outils complets copiés sur les modèles industriels. Leur compétitivité tient à leur capacité à produire des outils bon marché et adaptés à la demande locale $(9,16,20)$. Ils ont des contacts fréquents avec les paysans et sont souvent eux-mêmes agriculteurs. Cet avantage leur permet d'adapter les outils aux besoins. Au Sénégal, par exemple, les artisans ont mis au point une mini-houe Sine pour la traction asine et une lame souleveuse en demi-lune. Pour limiter les coûts de production, ils utilisent de la matière d'œuvre de récupération et suppriment les accessoires que les paysans n'utilisent pas ou bien les simplifient $(16,36)$. Au Nord-Cameroun, les charrues artisanales sont en moyennes deux fois moins chères que les charrues industrielles. Cette économie des coûts de fabrication ne se fait pas forcément au détriment de la robustesse et de la qualité (16).

Pour les matériels sophistiqués, la fabrication artisanale est problématique. Au Sénégal, l'entretien des systèmes de distribution des semoirs pose problème (9). Les pignons en bronze adaptés sont fragiles et mal ajustés, les disques fondus en aluminium ne permettent pas une distribution régulière. Au Cameroun, peu d'artisans parviennent à entretenir les moyeux des roues métalliques de grand diamètre des chars à boeufs. Les paysans sont souvent contraints d'utiliser de très vieux outils peu performants. L'origine du problème tient au manque de formation technique des artisans qui ont presque toujours été tenus à l'écart des programmes de mécanisation (20).

\section{Herbicides et repli du labour mécanique}

Au Nord-Cameroun, l'essor des herbicides s'est accompagné d'un recul du labour mécanique (31). Entre 1999 et 2003, la surface cotonnière traitée aux herbicides est passée de 50 à 71 p. 100, tandis que les surfaces labourées sont passées de 70 à 53 p. 100 (23). Dans la même optique, de plus en plus de paysans abandonnent les charrues lourdes (11 inches) pour des charrues légères (9 inches) et effectuent des labours superficiels combinés aux herbicides (36). Au sud du bassin cotonnier, 60 p. 100 des producteurs ont remplacé le labour mécanique par un désherbage au glyphosate suivi d'un épandage d'herbicide sélectif du coton et du maïs (23). Cette pratique qu'ils ont dénommé le « labour chimique » permet le semis direct et précoce en réduisant le temps de préparation d'un hectare à quelques heures seulement au lieu des trois à quatre jours en culture attelée. Pour les locataires d'attelages, c'est une formule très attrayante.

La simplification du labour a été observée dans d'autres situations où les herbicides ne sont pas en cause, comme dans l'est du Burkina Faso, où un tiers des producteurs pratiquent un pseudo-labour limité à la ligne de semis avec un corps butteur débarrassé de ses ailes (15), et dans la région de Guider au Nord-Cameroun. C'est surtout la rapidité d'intervention qui est alors visée. Dans ce cas, la fonction du labour est la préparation du lit de semences en facilitant l'infiltration des premières pluies.

\section{Vieillissement des semoirs et dégradation des semis}

Au Sénégal, la majorité des paysans utilisent les semoirs acquis entre 1960 et 1980. De nombreux semoirs hors d'usage ne sont plus remplacés $(3,9)$. On ne comptait plus qu'un semoir pour 8 ha contre un pour 6,5 ha en $1987(3,12)$. L'usure provoque une dégradation des semis (manquants, brisures...) et des pannes intempestives. Des essais chez des paysans (9) ont montré qu'avec un semoir neuf et des semences sélectionnées la densité de semis est satisfaisante et régulière (111 $600 \pm 4200$ pieds/ha), alors qu'avec un semoir paysan et des semences paysannes elle est faible et irrégulière (82 $500 \pm 35000$ pieds/ha). Pour l'arachide, cette dégradation du semis est aussi liée à la désorganisation du marché des semences. L'accès à des semences bien calibrées devient de plus en plus difficile. Les conditions d'utilisation des semoirs ne sont plus suivies (choisir un bon disque ne veut plus rien dire...).

\section{Essor de la mécanisation du désherbage dans les zones subhumides}

Dans ces zones, l'essor des équipements et des pratiques de désherbage mécanique a été conditionné par la conjonction des éléments suivants (31): i) un taux d'équipement local en charrues satisfaisant ; ii) un niveau de pression foncière suffisamment élevé pour conduire à la pratique de la culture continue; iii) des sols suffisamment filtrants et pas trop argileux pour ne pas provoquer l'enlisement des outils.

Lorsque ces conditions sont réunies, les producteurs passent progressivement d'une stratégie agricole extensive basée sur l'utilisation du labour à la charrue, à une stratégie plus intensive au sens où, l'espace se réduisant, il convient d'intensifier par le travail pour maîtriser les adventices sur une surface cultivable qui n'est plus extensible, et avoir recours aux engrais chimiques et organiques.

$\mathrm{Au}$ Nord-Cameroun sur les anciens fronts pionniers proches de Garoua, la densité de population est passée de moins de 10 à environ 40 hab. $/ \mathrm{km}^{2}$ entre 1990 et 2000 . Sur le même pas de temps, le parc des outils de sarclage a été multiplié par quatre et la surface cotonnière sarclée mécaniquement est passée de 30 p. 100 à plus de 60 p. 100 (23). 


\section{Recomposition des synergies agriculture élevage et transport attelé}

Autrefois, les systèmes de production étaient peu monétarisés et souvent caractérisés par une dissociation marquée entre l'élevage et l'agriculture dans des espaces où s'exerçait une faible pression sur les ressources naturelles.

Depuis une dizaine d'années, ces zones sont en proie à des dynamiques très fortes. Dans un contexte économique incertain en constante mutation (variations erratiques des prix agricoles, réduction de l'offre de services...) et pour saisir les opportunités qui se présentent (débouché garanti du coton, essor de la demande urbaine en denrées agricoles...), les producteurs mettent en place des systèmes de production mixtes combinant l'agriculture et l'élevage et diversifient leurs productions (lait, porcs, maraîchage, culture de bas-fonds, charbon de bois...). Ces nouvelles activités sont exigeantes en aliments pour le bétail et matières fertilisantes. Les revenus sont mieux sécurisés, mais la compétition sur les ressources naturelles est accrue (terres agricoles, pâturages, eau) et exacerbe les tensions et conflits entre communautés villageoises.

En conséquence, les écosystèmes ruraux atteignent progressivement des seuils critiques (les jachères tendent à disparaître, la fertilité des sols baisse, les ressources pastorales sont insuffisantes et se dégradent). Lorsque ces seuils sont dépassés, les agriculteurs et les éleveurs sont confrontés à des difficultés pour entretenir la fertilité des sols et pour satisfaire les besoins du bétail en fourrage. Dès lors, ils cherchent à mieux valoriser les biomasses fourragères et fertilisantes produites et/ou recyclées sur l'exploitation (fumier, compost, poudrette, résidus de cultures, sons...) ou bien prélevées sur les ressources communautaires (forêts, vaines pâtures...). C'est ainsi que se mettent en place des formes de complémentarités renouvelées entre productions animales et végétales.

Tant que la jachère et les défrichements restent possibles (faible densité de population rurale), ces relations agriculture élevage se limitent le plus souvent à la culture attelée et secondairement au transport (17). Lorsque la densité de population dépasse un certain seuil (généralement plus de $40 \mathrm{hab} . / \mathrm{km}^{2}$ dans les zones de savanes), on constate un passage progressif à la culture continue. La disparition des jachères et des friches induit de nouveaux modes de gestion de la fertilité (6) : recours systématique aux engrais chimiques sur coton et maiis ; fumure organique sur coton, maïs et sorgho ; rotations et associations céréales/légumineuses. Lorsque le cheptel autochtone prend de l'importance, on constate un développement des pratiques de stockage des résidus agricoles, de production de culture à usage alimentaire et fourrager (niébé fourrager, sorgho koidawa ; 7).

La valorisation de ces biomasses implique divers transports entre les « champs » et l'exploitation (stockage des fourrages et des litières, épandage de la fumure organique). Le transport attelé prend alors beaucoup d'importance. C'est notamment ce qui a été observé dans le bassin arachidier du Sénégal, au Mali Sud et dans une moindre mesure dans l'Extrême-Nord Cameroun. Ainsi, les producteurs stockent une partie des fourrages dès la récolte, puis ouvrent leur champ à la vaine pâture et au parcage, puis récupèrent en fin de saison sèche les résidus pour les litières et le compostage $(5,9,33)$.

CONSEQUENCES SUR LA DURABILITE DES SYSTEMES DE PRODUCTION ET PROPOSITIONS D'AMELIORATION

\section{Conséquences sur la viabilité, la vivabilité et la reproductibilité des systèmes de production}

Le tableau II présente les conséquences de l'évolution des pratiques de traction animale sur les composantes de la durabilité des systèmes de production. Pour une même innovation, on peut constater que certaines conséquences sont positives (accroissement de la flexibilité et de l'ergonomie, réduction des dépenses, diversification des revenus...), d'autres négatives (risques de sélection d'adventices, augmentation de certaines charges, baisse des rendements...) et certaines sans effet. Dans une perspective de gestion technique prévisionnelle de l'exploitation, il paraît donc indispensable de procéder à cet inventaire des gains et des pertes liés à une innovation, afin d'évaluer le coût d'opportunité du changement technique avant et après l'adoption (1).

\section{Propositions d'amélioration sur les aspects liés à la vivabilité}

Avec les équidés, et les ânes en particulier, la réduction de pénibilité est limitée car leur force de traction est modeste. Pour accroître leur puissance et leur endurance, il faut rechercher des solutions pour améliorer les harnachements et l'alimentation. La capacité de travail des différentes espèces et leur adaptation physiologique à l'effort sont des sujets désormais bien documentés (24). Des recommandations alimentaires ont été élaborées pour ajuster la ration de l'âne à l'effort et une grille de notation de l'état corporel des ânes a été mise au point pour piloter l'affouragement et notamment repérer les états critiques (32). Au sujet des harnachements, des modèles de bricoles améliorées (26) et des modes d'attelage par paires ont été testés (figure 2). Ces travaux s'appuient sur des études des caractéristiques morphologiques des ânes (8).

Concernant l'efficacité du désherbage mécanique, elle peut être améliorée par une intervention précoce et répétée (14), et des harnais adaptés au passage entre des rangs de cultures (28). Testé sur coton, chez des paysans du Nord-Cameroun, le sarclage mécanique précoce réduit par trois la durée d'intervention à l'hectare par rapport aux pratiques paysannes (21 à $7 \mathrm{j} / \mathrm{ha}$, finitions comprises). Le jouguet monobovin diffusé au Nord-Cameroun (figure 2) permet une conduite aisée et précise de l'attelage dans les rangs de culture (diminution des piétinements, virages plus rapides...).

S'agissant des techniques de transport, l'effort de développement doit porter sur l'Afrique centrale et les zones subhumides qui accusent un important retard en la matière. Un transfert de techniques sud-sud pourrait être envisagé avec l'introduction des nombreux modèles d'Afrique de l'Ouest de charrettes légères et de tombereaux asins, en s'appuyant sur les réseaux de forgerons locaux. Dans les zones de montagnes d'Afrique centrale, le portage est une technique qui mériterait d'être développée. L'expérience de l'Ethiopie est très éclairante en la matière et source d'inspiration pour des propositions techniques (25; figure 2 ).

Pour réduire la fréquence des pannes et de la casse des outils, il faudrait des programmes de formation des artisans forgerons. Les bons artisans sont demandeurs de mise en place de procédures de certification pour labelliser leur production et les protéger des productions douteuses (36). Une grille de contrôle technique des charrues bovines basée sur 14 points de vérification a été expérimentée et a permis de montrer que les artisans aussi peuvent produire des outils de bonne qualité (36).

\section{Propositions d'amélioration sur les aspects liés à la viabilité économique}

Pour améliorer la production et les rendements avec les techniques de cultures associant mécanisation et herbicides, plusieurs pistes ont été expérimentées. La technique de travail du sol en sec au coutrier sur la ligne de semis augmente l'infiltration des premières pluies et ameublit le sol, ce qui favorise la germination et l'enracinement (figure 2). Les resemis sont moins nombreux et les rendements meilleurs (100 
à $200 \mathrm{~kg} / \mathrm{ha}$ de plus pour le sorgho par rapport au semis direct pratiqué par les paysans). Cette opération est rapide puisque le sol n'est travaillé que sur la ligne de semis, soit tous les $80 \mathrm{~cm}$ pour le coton, et n'intervient pas en concurrence avec d'autres chantiers puisqu'elle est effectuée avant les premières pluies. Elle requiert une paire de bovins puissante et un sol ni trop sableux, ni trop argileux (30).

Dans la même optique d'augmentation de la production et du rendement, la technique du sarclage mécanique précoce et répétée permet une amélioration significative en réduisant les compétitions culture/ adventices pour l'eau et les éléments fertilisants. Pour les essais sur coton conduits en milieu paysan sur le cotonnier, l'amélioration du rendement a été de $170 \mathrm{~kg} / \mathrm{ha}$, soit 14 p. 100 environ par rapport aux pratiques de sarclage classique des producteurs (14).

S'agissant des augmentations de charges d'intrants pour l'agriculture et pour l'alimentation animale, un effort de formation et de conseil doit être conduit au niveau des producteurs et de leurs organisations. Les résultats de la recherche pour l'alimentation des bovins et des ânes de trait en zone tropicale doivent être traduits en recommandations pratiques $(18,29)$. Une documentation technique sur les herbicides et les autres intrants agricoles doit être largement

Tableau II

Evolutions des pratiques de traction animale et conséquences sur la durabilité des systèmes de production

\section{Conséquences sur la :}

Evolutions des pratiques

Vivabilité
(pénibilité, qualité de
vie, charge mentale)

Diversification des Location modes d'accès à

la traction animale

Dvpt des
attelages
légers

Risque d'accumulation

de travaux en retard

Force de travail augmentée :

Modérément avec un cheval

Peu avec un âne

\section{Viabilité économique (production, rentabilité)}

Augmentation des surfaces cultivées (par rapport au

Surendettement évité Extension des surfaces cultivées (par rapport au travail manuel) Dvpt d'activités génératrices de revenu travail manuel)
Adaptation des matériels par les forgerons, simplification et réduction des coûts
Amélioration ergonomie Augmentation casses et pannes (matière d'œuvre de récupération)
Réduction dépenses

d'équipement

Augmentation dépenses

de réparation

Baisse rendements possible

(dégradation semis

si mauvais matériel)
Economie d'équipement Augmentation des charges d'intrants
Baisse des rendements possible

\section{Reproductibilité \\ (effets sur les \\ ressources naturelles)}

Aucune

Aucune

Auc

$\begin{array}{ll}\text { Essor des herbicides et repli } & \begin{array}{l}\text { Gain de temps et } \\ \text { du labour mécanique }\end{array} \\ \text { (sur coton et maïs) } & \text { de cycle }\end{array}$

(sur coton et maïs) de flexibilité en début
Essor des herbicides et repli
Réduction des pics de minéralisation de la matière organique

Risque de sélection de flore d'adventices en cas de sous-dosage

$\begin{array}{ll}\text { Vieillissement des semoirs } & \text { Pannes et pertes de temps } \\ \begin{array}{l}\text { Extension du désherbage } \\ \text { mécanique }\end{array} & \begin{array}{l}\text { Gain de temps important } \\ \text { en milieu de cycle }\end{array}\end{array}$

Dégradation des semis et baisse de la production

Augmentation des rendements (concurrences cultures/ adventices limitées)

\section{Aucune}

Risque d'aggravation de l'envahissement par certaines adventices dominantes (ex : Commelina spp.)

Entretien de la fertilité de la terre et soutien au développement du cheptel agriculture élevage

par le développement

du transport attelé
Réduction de la pénibilité

Entretien de la fertilité de la terre avec effet sur les rendements
Réduction de la morbidité des animaux en saison sèche grâce à l'affouragement 


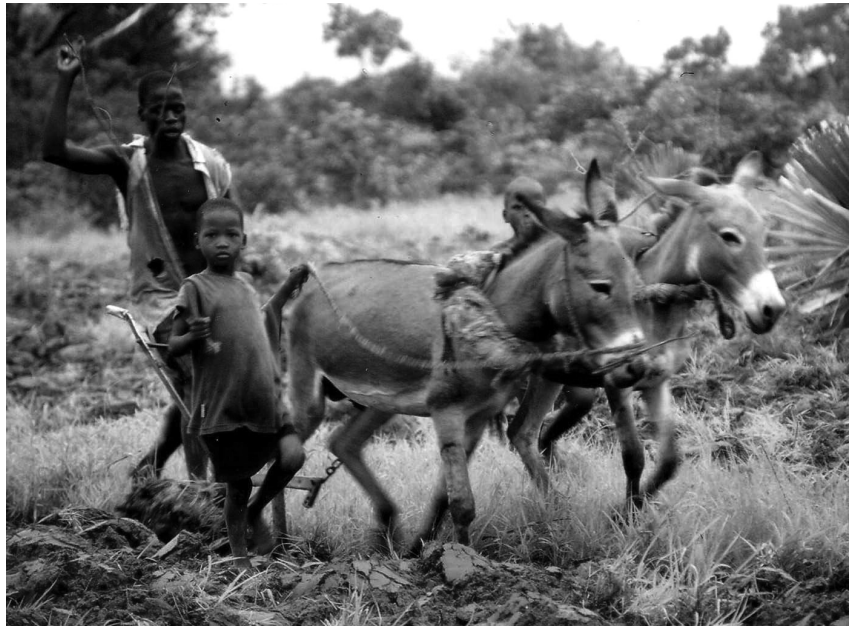

Labour à la paire d'ânes (M. Havard)

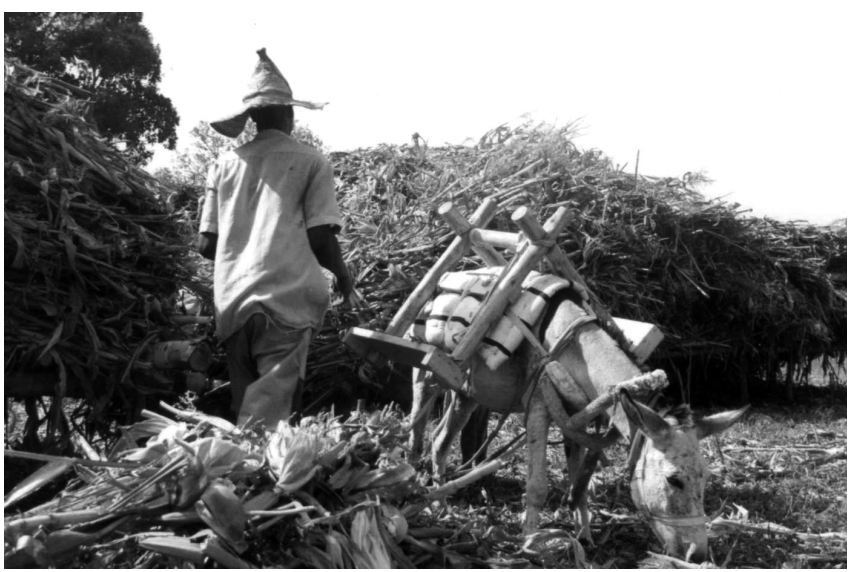

Bât asin expérimenté (E. Vall)

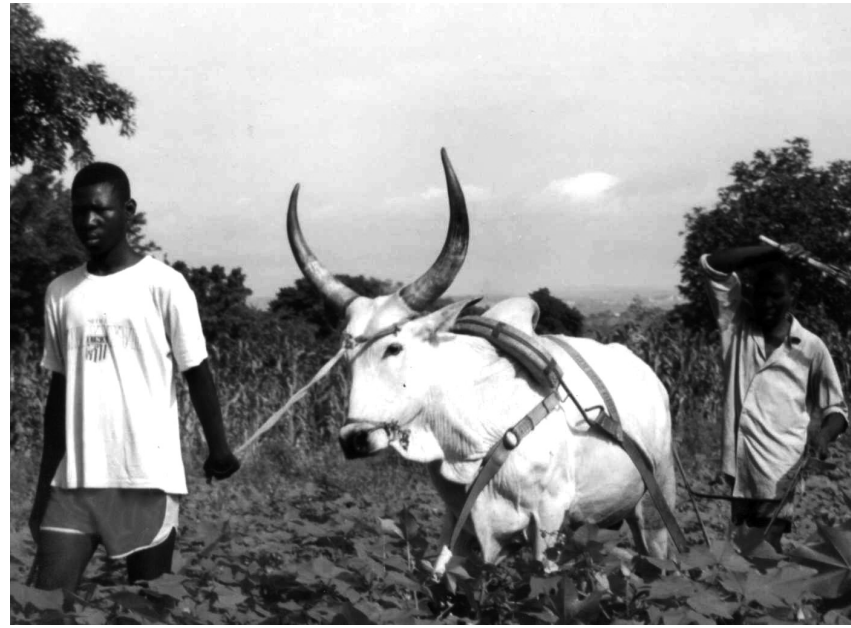

Sarclage au jouguet monobovin (E. Vall)

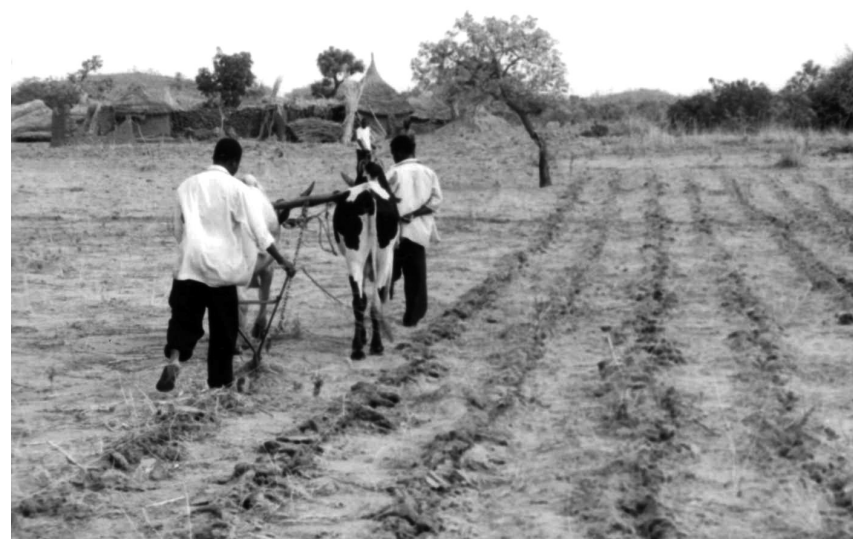

Travail du sol en sec au coutrier (E. Vall)

Figure 2 : techniques de traction animale expérimentées au Nord-Cameroun.

diffusée pour améliorer leur utilisation, mais aussi pour attirer l'attention des producteurs sur les risques environnementaux liés à un mauvais usage de ces produits.

\section{Propositions d'amélioration sur les aspects liés à la reproductibilité}

L'essor des herbicides et le recul du labour, mais aussi la diffusion du sarclage mécanique augmentent les risques de prolifération et de sélection de flores d'adventices lorsque les techniques ne sont pas bien maîtrisées (sous dosage des herbicides, mauvaise conception des outils...). Il faut intervenir à la fois sur les composantes et sur la globalité des itinéraires techniques concernés. Pour les herbicides, il est nécessaire de former les paysans sur les modes d'action et les modalités d'applications de ces produits (31). Or, force est de constater que les fiches techniques de la recherche et du développement ne sont pas assez diffusées. Pour la conception des outils de sarclage, leur efficacité au désherbage dépend de la forme des dents, de l'angle d'entrure des socs et de la forme des étançons afin d'optimiser l'extirpation et de limiter le sectionnement et l'enfouissement des adventices (27).

Dans une optique d'amélioration des itinéraires techniques, il s'agit de résoudre les difficultés d'enracinement dans un sol non travaillé et une moins bonne maîtrise des adventices, lorsque les paysans abandonnent totalement le labour au profit des herbicides (labour chimique). Deux opérations de travail du sol peuvent compléter l'itinéraire technique classique du labour chimique sur cotonnier (figure 3). Le travail du sol en sec au coutrier favorise l'infiltration des pluies et par conséquent la germination et l'enracinement du cotonnier. Le scarifiage exacerbe la germination d'adventices dominantes, comme Commelina spp., ce qui multiplie l'efficacité de l'herbicide. Par rapport au labour chimique sans travail du sol, on constate une meilleure levée, une meilleure maîtrise des adventices et une augmentation de production de $250 \mathrm{~kg} / \mathrm{ha}$ de coton (30). L'objectif de gain de temps et de desserrement des calendriers agricoles en début de campagne, visé par ces techniques de culture simplifiées, est maintenu, car les travaux du sol sont étalés dans le temps. De plus, ce sont des opérations rapides, dont la durée cumulée est inférieure à celle d'un labour d'environ 25 p. 100. Notons que dans cet itinéraire technique, les différentes fonctions du labour se trouvent décomposées.

Pour le renforcement des synergies entre l'agriculture et l'élevage, notamment dans les situations de forte compétition sur les ressources, il s'agit d'améliorer les capacités de gestion des producteurs pour les biomasses participants aux relations agriculture élevage, tant au niveau individuel (exploitation) que collectif (communauté villageoise). En s'appuyant sur un diagnostic systémique à l'échelle d'un village (typologies, étude des pratiques et de leurs déterminants), la recherche et le développement se doivent de proposer des solutions intégrées à trois niveaux : le système 


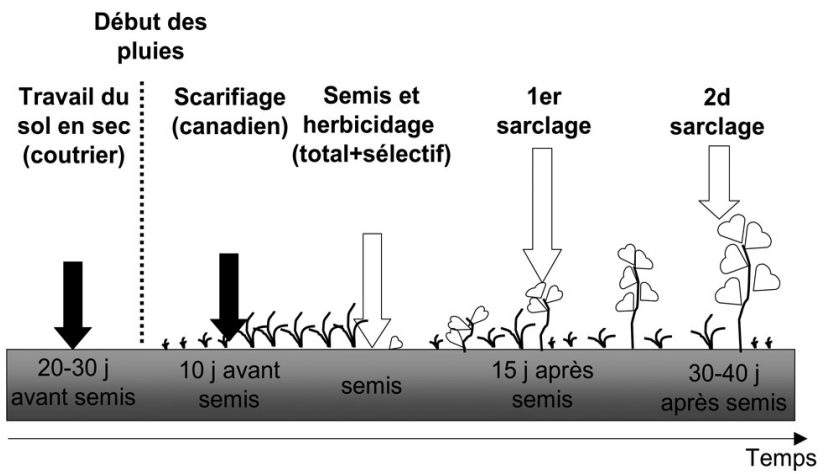

Figure 3 : une amélioration du "labour chimique" pour le cotonnier.

technique, tout d'abord, pour augmenter la production, réduire les pertes, améliorer la valorisation des biomasses et sensibiliser les producteurs sur l'importance du transport ; l'exploitation agricole, ensuite, pour accompagner les changements de pratiques, en formalisant le raisonnement du producteur en termes de coût d'opportunité (bilan des gains et des pertes avec ou sans innovation compte tenu de la situation de l'exploitation) ; la communauté villageoise, enfin, pour tout ce qui concerne la maîtrise des feux, le droit de vaine pâture, l'exploitation des ressources fourragères naturelles (fourrages ligneux, aquatiques...), c'est-à-dire la gestion concertée et équitable des ressources collectives (6).

\section{CONCLUSION}

Dans les systèmes de culture des zones semi-arides et subhumides, la traction animale occupe toujours une place centrale dans les stratégies des producteurs en renforçant la productivité du travail à plusieurs étapes déterminantes du calendrier agricole : préparation des terres, semis, désherbages, transport des récoltes et des résidus de culture. Cependant, les taux d'équipements en matériel de transport restent bien souvent insuffisants pour permettre une amélioration notable de ces synergies entre l'agriculture et l'élevage.

Ces dix dernières années, le désengagement des Etats du secteur rural et le démantèlement des services d'appui à l'agriculture ont modifié les conditions d'accès et d'entretien pour les animaux et pour les outils. Cette évolution a bénéficié à l'artisanat du fer. Mais pour les outils chers et difficiles à réaliser, les durées d'utilisation ont augmenté, le parc a vieilli, les outils sont usés et moins performants. L'augmentation de la pression foncière, ayant conduit à l'extension des zones de culture et à l'augmentation du cheptel, a fortement accru la compétition sur les ressources en terres agricoles et en parcours. Lorsque les communautés parviennent à s'organiser pour l'usage individuel et collectif de ces ressources, on constate un renforcement de l'intégration de l'élevage et de l'agriculture (valorisation de la fumure animale et des résidus agricoles fourragers) et l'apparition de pratiques d'intensification par le travail (semis mécanique...). Enfin, la diffusion des herbicides conduit à une simplification des techniques de préparation des terres avant le semis et à une réorganisation des calendriers agricoles des paysans. Dans les régions où la percée des herbicides est significative, on constate un recul des surfaces labourées à la charrue.

Les conséquences agronomiques de l'évolution des pratiques de traction animale sur la durabilité des systèmes de production sont parfois positives lorsqu'elles contribuent à améliorer la flexibilité du travail, et la production et les revenus financiers des ménages, et à augmenter la rentabilité de l'attelage (diversification des usages); elles vont dans le sens d'un renforcement des synergies agriculture élevage. Mais lorsque l'évolution est simplement guidée par la volonté individuelle de valoriser une opportunité de marché à court terme qui ne s'inscrit pas dans un schéma global de développement du territoire villageois, la durabilité de l'agriculture peut être compromise (c'est par exemple le cas avec des techniques de culture simplifiées mal maitrisées). La recherche doit proposer des méthodes visant à renforcer les capacités de gestion des producteurs afin de sécuriser les options qu'ils mettent en œuvre individuellement et collectivement (10).

Face à cette nouvelle donne, les agronomes ont l'obligation de renouveler leurs modes d'intervention et leurs pratiques de recherche concernant la traction animale (34). Les travaux antérieurs visaient la mise au point des équipements et des normes d'utilisation. Aujourd'hui les objectifs visent autant la multifonctionnalité de la technique (culture attelée, transport, sécurisation financière des ménages) que les conditions de pérennisation des services d'appui à la traction animale. L'intervention des agronomes se conçoit dans un cadre transdisciplinaire et dans une perspective d'innovation en partenariat avec les acteurs qui composent le système de services d'appui à la traction animale (vétérinaires, forgerons, banquiers, services d'appui/conseil...).

\section{BIBLIOGRAPHIE}

1. BROSSIER J., CHIA E., MARSHALL E., PETIT M., 1997. Gestion de I'exploitation agricole familiale. Dijon, France, Enesad-Cnerta, 215 p.

2. CUVIER L., 1999. Etude des pratiques et des stratégies paysannes de traction animale dans la zone cotonnière du Nord-Cameroun : cas du terroir de Mafa-Kilda. Montpellier, France, Cirad-emvt, 82 p.

3. DjimTOlOuM E., 2000. Etudes des pratiques et des stratégies paysannes en matière de traction animale dans le sud du bassin arachidier du Sénégal : cas du village de Keur Bakary - Communauté rurale de Kaymor. Montpellier, France, Cnearc, 92 p.

4. DONGMO NGOUTSOP A.L., 1999. Pratiques et stratégies paysannes de traction animale en zone cotonnière du Nord-Cameroun : cas du terroir de Mowo. Dschang, Cameroun, FASA, 121 p.

5. DUGUE P., 1998. Les transferts de fertilité dus à l'élevage en zone de savane. Agric. Dév., 18 : 99-107.

6. DUGUE P., VALL E., KLEIN H.D., ROLLIN D., LECOMTE P., 2004. Evolution des relations entre l'agriculture et l'élevage dans les savanes d'Afrique de l'Ouest et du Centre. Ol., Corps gras, Lipides, 11 : 20.

7. DUGUE P., VAlL E., CATHALA M., MATHIEU B., OLINA J.-P., SEUGE C., 2004. Les paysans innovent, que font les agronomes? Le cas des systèmes de culture en zone cotonnière du Cameroun. In : $3^{\text {e }}$ édn Journées Olivier de Serres, Agronomes et Innovation, Le Pradel, France, 21 p.

8. EBANGI A.L., VALL E., 1998. Phenotypic characterization of draft donkeys within the Sudano-Sahelian zone of Cameroon. Revue Elev. Méd. vét. Pays trop., 51 : 327-334.

9. FALL A., NDAO T., DIAGNE K., MBODJ M., 2001. Action thématique programmée sur la traction animale au Sénégal : rapport de synthèse. Bambey, Sénégal, Isra, 76 p.

10. FAURE G., DUGUE P., BEAUVAL V., 2004. Conseil à I'exploitation familiale. Expériences en Afrique de l'Ouest et du Centre. Guide pratique. Paris, France, Gret-Cirad, 127 p.

11. HAVARD M., 1988. Les conclusions des expérimentations (19501985) sur les semis en culture attelée des principales espèces cultivées. Mach. agric. trop., $101: 11-51$.

12. HAVARD M., FAYE A., 1988. Eléments d'analyse de la situation actuelle de la culture attelée au Sénégal : perspectives d'études et de recherches. In : Starkey P., Ndiamé F. éds, Animal power in farming systems. Esborn, Allemagne, Gate, GTZ, p. 241-252.

13. HAVARD M., LE THIEC G., VALL E., 1998. Stock number and use of animal traction in Sub-Saharan French-speaking Africa. Agric. Mech. Asia, Afr. Latin Am., 29: 9-14.

14. HUGUENOT S., 2001. Test d'une innovation : le sarclage mécanique précoce à Mafa Kilda (Nord-Cameroun). DESS Gsaspzt, Paris XII , Créteil, France, $59 \mathrm{p}$.

15. ILBOUDO I., 2001. Etude socio-économique des pratiques agricoles et évaluation du passage de la culture manuelle à la culture attelée dans la Tapoa, Burkina Faso: cas des zones de Botou et Boaguidigou. BoboDioulasso, Burkina-Faso, IDR, 96 p. 
16. KEMTSOP TCHINDA G.A., 1999. Fabrication des agro-équipements de traction animale dans la province du nord du Cameroun : place et rôle de l'artisanat du fer. Dschang, Cameroun, Fasa, 94 p.

17. LANDAIS E., LHOSTE P., 1990. L'association agriculture-élevage en Afrique intertropicale: un mythe techniciste confronté aux réalités du terrain. Cah. Sci. Hum., $26: 217-235$.

18. LAWRENCE P.R., PEARSON R.A., 1999. Feeding standards for cattle used for work. Edinburgh, Scotland, UK, University of Edinburgh, CTVM, $59 \mathrm{p}$.

19. LE THIEC G. coord., 1996. Agriculture africaine et traction animale. Montpellier, France, Cirad, 355 p. (Coll. Techniques)

20. LE THIEC G., HAVARD M., 1996. Les enjeux du marché des matériels agricoles pour la traction animale en Afrique de l'Ouest. Agric. Dév., 11 : 39-51.

21. LY C., FALL B., CAMARA B., NDIAYE C.M., 1998. Le transport hippomobile urbain au Sénégal. Situation et importance économique dans la ville de Thiès. Revue Elev. Méd. vét. Pays trop., 51 : 165-172.

22. POURTIER R., 2003. Les savanes africaines entre local et global: milieux, sociétés, espaces. Cah. Agric., $12:$ :213-218.

23. SODECOTON, 2002. Rapport semestriel (mai-octobre 2002). Campagne agricole 2002/2003. Garoua, Cameroun, DPA, Sodécoton, 70 p.

24. VALL E., 1996. Capacités de travail, comportement à l'effort et réponses physiologiques du zébu, de l'âne et du cheval au NordCameroun. Thèse Doct., Ensam, Montpellier, France, 418 p.

25. VALL E., ABAKAR O., 2001. Perfecting donkey saddles in the NorthCameroon savana zone. Agric. Mech. Asia, Afr. Latin Am., 32: 12-18.

26. VALL E., ABAKAR O., 2003. La bricole asine améliorée. Fiche technique n 9. N’Djaména, Tchad, Prasac, $2 \mathrm{p}$.

27. VALL E., ABAKAR O., ABOUBAKARY, TCHINSABHE P.I., 2003. La houe Attila: un outil de désherbage conçu pour la traction asine. Fiche technique $\mathrm{n}^{\circ}$ 14. N’Djaména, Tchad, Prasac, 2 p.

28. VALL E., ABAKAR O., KPOUMIE E., 2001. Mise au point de jougets monobovins pour la culture attelée dans la zone de savane du Nord Cameroun. Revue Elev. Méd. vét. Pays trop., 54 : 247-253.
29. VALL E., ABAKAR O., LHOSTE P., 2003. Adjusting the feed supply of draught donkeys to the intensity of their work. In: Pearson R.A., Lhoste P., Saastamoinen M., Martin-Rosset W., Eds. Working animals in agriculture and transport. EAAP Technical series No 6. Wagneningen, The Netherlands, Wagneningen Academic Publishers, p. 79-91.

30. VALL E., ABOUBAKARY, ABAKAR O., 2003. Le travail du sol en sec au coutrier. Fiche technique $n^{\circ} 17$. N'Djaména, Tchad, Prasac, 2 p.

31. VALL E., CATHALA M., MARNOTTE P., PIROT R., OLINA J.-P., MATHIEU B., GUIBERT H., NAUDIN K., ABOUBAKARY, PABAME TCHINSAHBE I., 2002. Pourquoi inciter les agriculteurs à innover dans les techniques de désherbage? Etat de la pratique et propositions de la recherche. In : Savanes africaines : des espaces en mutation, des acteurs face à de nouveaux défis, Garoua, Cameroun, 27-31 mai 2002, 19 p.

32. VALL E., EBANGI A.L., ABAKAR O., 2001. Mise au point d'une grille de notation de l'état corporel des ânes de trait au Nord Cameroun. Revue Elev. Méd. vét. Pays trop., $54:$ 255-262.

33. VALL E., DONGMO NGOUTSOP A.L., ABAKAR O., MEYER C., 2002. La traction animale dans le nouveau contexte des savanes cotonnières du Tchad, du Cameroun, et de la République centrafricaine. I. Diffusion de la traction animale et sa place dans les exploitations. Revue Elev. Méd. vét. Pays trop., 55 : 117-128.

34. VALL E., LHOSTE P., ABAKAR O., DONGMO NGOUTSOP A.L., 2003. La traction animale dans le contexte en mutation de l'Afrique subsaharienne: enjeux de développement et de recherche. Cah. Agric., $12: 219-226$

35. VALL E., ROESCH M., KLEENE P., WAMPFLER B., TRAORE A.D. 2003. Compte rendu de l'atelier de bilan et d'échanges sur les travaux de I'ATP au Burkina Faso. Montpellier, France, Cirad-emvt, 105 p.

36. VANDENDAEL L., 2000. La charrue des paysans du Nord-Cameroun : un outil, son utilisation et sa fabrication. Gembloux, Belgique, FUSAGx, $85 \mathrm{p}$.

37. VIDEAULT S., 2001. Analyse des besoins et des stratégies de financement de la traction animale des exploitations agro-pastorales de la province de la Tapoa. Burkina Faso - Pays Gourmanché. Paris, France, Istom, $119 \mathrm{p}$.

\section{Summary}

Vall E., Dongmo Ngoutsop A.L., Ndao T., Ilboudo I. Evolution of Draft Animal Power Practices and Consequences on Crop Systems Sustainability

In West and Central Africa savannah zone crop systems, draft animal power plays a major role by providing farmers with increased intervention means during key steps of the agricultural calendar: ploughing, seeding, weeding, transport. In the past ten years, three factors led to practices evolution: i) dismantling public agricultural services made purchasing and maintaining animal teams more difficult; ii) land pressure increase in rural zones reinforced crops and livestock integration, and induced practices based on work intensification (mechanized weeding...); iii) broad use of herbicides resulted in ploughing decline. Agronomic consequences of these changes on crop systems sustainability are sometimes positive (more work flexibility; production, and household income increases) and sometimes negative (marginal role of animal husbandry in fertility transfers, degradation of mechanical seeding, degradation of crop management sequences, deforestation...). Various ways to improve the technique of animal traction are proposed.

Keywords: Animal Power - Draft Animal cultivation - West Africa - Central Africa.

\section{Resumen}

Vall E., Dongmo Ngoutsop A.L., Ndao T., Ilboudo I. Evolución de las prácticas de tracción animal y consecuencias sobre la durabilidad de los sistemas de cultivo

En los sistemas de cultivo de las zonas de sabana de Africa del Oeste y central, la tracción animal ocupa un lugar determinante para el refuerzo de la capacidad de intervención de los productores durante las etapas clave del calendario agrícola: preparación de las tierras, cultivo, desherbado, transporte. Estos últimos diez años, tres factores han determinado las evoluciones de las prácticas: i) el desmantelamiento de los servicios de apoyo público a dificultado la adquisición y el mantenimiento de los atelajes, ii) el aumento de la presión territorial en zona rural a reforzado las sinergias agriculturacría e inducido a prácticas de intensificación mediante el trabajo (mecanización de los desherbados...), iii) la popularización de los herbicidas a provocado una regresión de la labor. Las consecuencias agronómicas de estas evoluciones sobre la durabilidad de los sistemas de producción son a veces positivas (mejora de la flexibilidad del trabajo, de la producción y de los ingresos financieros de los hogares), a veces negativas (marginalización del papel de la cría en los traspasos de fertilidad, degradación del cultivo mecánico, degradación de los itinerarios técnicos, deforestación...). Se sugieren proposiciones para el mejoramiento de la técnica.

Palabras clave: Energía animal - Cultivo a tracción animal Afrique Occidental - Afrique Central. 